\title{
GENERATION AND SEARCH METHODS IN DESIGN: DISCUSSION
}

\author{
KEN BROWN \\ Carnegie Mellon University, USA
}

\section{Introduction}

Design generation is the process of formulating design solutions within a design space. An early view of design as a search process was discussed by Simon (1969), in which knowledge can be modeled as a set of operators, and design problems modeled as goals; design is then the process of searching for sequences of operators which produce solutions to those goals. Depending on the nature of the operators, this process may involve the refinement and transformation of incomplete designs until a complete solution is obtained, or a traversal through a space of possible solutions occurs, or a combination of both. The process may be deterministic, in which the space is searched systematically, according to fixed procedures, or stochastic, in which probabilistic methods are used to sample the space. Design as search has been criticised as being too restrictive to capture the real nature of design. Smithers et al. (1994) argue that a large part of design is concerned with formulating the problem and discovering the relationship between the design goals and design knowledge, and thus that design is better captured by the term exploration. The session summarized here considered methods of formally describing and searching spaces in order to generate design solutions, and discussed the adequacy of search as a characterization of design.

Two papers were presented in this session. Brown and Cagan (1996) present some results on the use of shape annealing for searching large state spaces. Moves within a space are selected probabilistically, where a move may include backtracking to an arbitrary height. The result of each move is evaluated, and is then either accepted or rejected with a probability derived from the evaluation. The algorithm converges to good solutions. The paper presents evidence that both the evaluation function and the details of the backtracking moves are important to the algorithm's success. ZozayaGorostiza and Estrada (1996) also investigate searching large spaces, although this time using genetic algorithms, which apply moves probabilistically to populations of designs. Again designs are evaluated, and 
multiple designs are selected to remain in the population. The paper demonstrates that both domain-specific heuristics and search in the meta space are important factors in the algorithm's performance.

These two papers have many features in common. Both considered large spaces, for which systematic search methods were assumed to be unsuitable; the ability to evaluate designs or states in the space was assumed; moves within the space were selected using random or probabilistic methods; and all three relied on statistical convergence to settle on good designs. Brown and Cagan's method considered only one candidate at a time, but ensured diverse paths through the space were considered by only gradually introducing stability as the search progressed. Zozaya and Estrada's method ensured diversity through maintaining multiple options throughout the search.

The discussion which followed was structured around three questions arising from these presentations:

1. Is the notion of random generation followed by evaluation and selection an adequate characterization of design?

2. Are there other methods of searching large design spaces?

3. Is modifying existing algorithms to suit particular problems a better approach than recoding the problem descriptions?

The text that follows does not strictly adhere to this structure, but reflects the flow of the discussion.

\section{Design as Random Generation and Selection}

The papers in the session presented methods in which design consisted of a series of more or less random moves to produce new states, interleaved with evaluation and selection. Is this a reasonable view of design? Firstly, it seems far from human design practice, in which steps are chosen with specific goals in mind. This raised the question of whether design is a human activity, or a more general phenomenon of which human design happens to be the best example. Secondly, assuming this approach is design, why should it be considered? One of the main reasons is that it is relatively easy to implement in computer systems, as it requires very little domain-specific design knowledge. There is no need to partition the search spaces, or to encode routines for deciding which options should be pursued in which circumstances. Once the space is described in terms of primitive elements and legal operators, generation is knowledge-free. All the knowledge is embedded in the evaluation function (and thus is only concerned with the designed artifacts and not the structure of the space used to create them). 
This approach views design as more of a mathematical optimization process than a human-centered artistic endeavour.

The approach above is dependent on the assumption that the knowledge for evaluating designs is available. In many design domains, and in architecture in particular, this is not necessarily true. A distinction has to be made between book-keeping evaluation, in which a design is assessed against specific well-defined criteria-for example, conformance to building standards, fire codes, safety regulations-and performance evaluation, in which a design is assessed for its likely performance in its environment - for example, its aesthetic appeal. The distribution of evaluation criteria between these two categories will have a big impact on the effectiveness of computerbased design tools.

As the randomly-generate-and-test methods lose the purposive feel of design, the question arises as to whether any generative methods can be intent-driven: instead of simply randomly generating a change, assessing its effect, and then deciding whether to accept it, is there a method of proposing synthesis moves oriented towards meeting a goal? Applying a pre-filter to the available moves before generating a change simply brings an implicit, approximate evaluation into play earlier in the process. Indexing or classifying operators according to the expected goals they will contribute to allows move selection to be based on intent, but the problem then becomes one of establishing a useful classification scheme. One type of approach which, it was suggested, does capture this intent-driven aspect of design is that found in case-based and analogical reasoning. Here, a library of known designs is searched and compared to the existing problem. Particular designs are selected, based on their perceived match with the problem. Modifications (moves in a design space) are then made to these designs in order to obtain a full solution to the given problem. Thus the design intent (the problem description) is used to select a set of seed designs. Selecting the appropriate modifications may still require the same approaches as above, but now a localized area of the design space has been selected for investigation.

Note that if the knowledge of the search space is available, then better solutions would probably be found more easily by using intent-based selection of the moves. However, this knowledge is not always available or easy to code, nor is it general. In that case, more general methods, in which all the knowledge is removed from the generation and put into the evaluation may be preferable. Machine learning may be applied to these systems in order to improve their performance on the specific problems, and the resulting move selection techniques would then correspond to intentbased synthesis. 


\section{Design as Search}

The second main area of discussion was the issue of whether design can be adequately characterized as search at all. Again, this hinged in part on the issue of whether human behaviour defines design. A number of points were raised which cast doubt on the adequacy of search as a description of the human design process. It was asserted that architectural designers, for example, tend to pursue a single idea, pushing it to conclusion, and forcing the idea to fit the requirements (or forcing the requirements to fit the idea). If this is the case, the process should not be described as search, as other options are simply not considered. Allied to this is the notion that good design follows from an inspiration, or a "great idea", and that good designs result from adapting that good idea to the present circumstances, rather than vaguely searching through the range of all logical options.

A number of other objections, more specific to formal design, were also raised. There is, in general, no well-defined path from function to form, contrary to the underlying assumption of many prescriptive design methods, and thus design is a matter of choice and judgement, rather than a search for the single answer implied by the problem statement. As discussed in the previous section, designs are frequently not quantifiable, and so search, requiring definite criteria against which different states are compared, cannot be carried out. Even if the designs can be evaluated, the requirements of the problem frequently change, either as a result of finding that the specifications are inconsistent or overly restrictive, or through explicit changes in the requirements or the environment during the process, or through discovering that the problem is under-specified, and that choices have to be made regarding which particular paths will be pursued. Design also involves the phenomenon of emergence: the re-interpretation of a representation producing structures which were not explicitly intended or created, and the use of these emergent structures in later design decisions. Standard search methods give little support for emergence, using fixed representations and interpretations throughout the process. This highlights the need for schemas which can be adapted dynamically as design proceeds.

Contrary to the flow of the above discussion, the question was raised as to whether there can be anything other than search in computer-based design. Almost every proposed approach can be reduced to search, usually by moving up a conceptual level. For example, instead of representing a design task as a problem with changing requirements, we could represent it as a search through a space of (requirement, design) pairs. However, if everything can be reduced to search, it is then doubtful that reducing processes to search is useful for understanding design. Although characterizing a problem as search might make it easier to implement as a computer system, 
it hides the difference between different approaches, and obscures their significant characteristics.

Finally, the point was raised that even if all the above objections are accepted, and search is not an adequate characterization of design, that does not mean that search should be abandoned as a tool-many specific design problems can be characterized as search, and many of the alternative approaches would require search as significant sub-processes.

\section{Research in Search-Based Design}

The third area of discussion during the session was the topic of research methods in search-based design. Firstly, the question was raised as to whether it is advisable to modify existing algorithms (as in the two presentations) to improve results on specific problems, or whether it is better to search for a better representation of the problem, allowing the standard algorithms to be applied successfully. The response was that if the problems are generic, then there is an advantage to modifying the algorithms. Two approaches are possible: designing better algorithms, or designing algorithms which are adaptive, varying their operation according to the specifics of the problem. Related to this topic, it was suggested that as we gather more knowledge on which algorithms perform best on which particular types of spaces, and at what phases of the design process, we should be progressing more towards hybrid algorithms, which start with one method, and then switch to another as we move to a different phase.

A more basic discussion point concerned the purpose of research into design methods. Our efforts should not be restricted to current design practice, but should principally be aimed at producing better designs. Of course, this may well involve providing better support for current practice, but it may also involve radically different methods, either in producing autonomous design systems, or using computer-based tools to change the way in which designers design. For example, it was mentioned above that architectural designers typically only pursue a single idea. Why is this? Is it because this is the best way of producing good designs, regardless of the support available, or is it a result of the limitations of human information management? It could be that managing multiple alternatives to any significant level of detail, including diagrams, models, evaluations, and ramifications is too complex a task for unsupported humans, but if support for these aspects was provided, and all the information made readily available, the standard design process might change from pursuing a single option to pursuing multiple options. Such questions need to be investigated, in order to direct future research towards the goal, as stressed above, of producing better designs. 
As a related point, we should consider whether or not "great idea" design is a good exemplar for our research. On a number of occasions during the discussion approaches were evaluated by comparing them to the approaches used to design well-known objects-for example, the Sydney Opera House. It is not clear that such examples provide a good focus for design computing research. In many ways, the successes or failures of these projects are discussed in terms of their uniqueness, the way in which they shift the bounds of current taste, and their innovative approach, and not necessarily in terms of how well they meet their original purpose. These criteria depend on an understanding of human reactions to buildings, human preferences, and attitudes to art and aesthetics. If our aim is to produce better designs, then our efforts should perhaps be directed towards more commonly encountered design tasks. Research would thus be centered on increased automation of design taskś which can be readily evaluated, and on generating alternatives and providing bookkeeping support for these more speculative tasks.

Finally, it is worth noting the seeming change in focus of design research between the workshop discussed here and the previous one in the series (Gero and Tyugu, 1994). In that previous workshop, there were six papers explicitly concerned with generative systems (Carlson, 1994; Heisserman and Woodbury, 1994; Shih and Schmitt, 1994; Brown et al., 1994; Andersson, 1994; Cagan and Mitchell, 1994). All six of them considered grammatical systems in particular. All except Cagan and Mitchell were largely concerned with presenting the spaces and the formalisms, with little concern for how those spaces would be searched. In the current workshop, there were five papers explicitly concerned with generation and search (the first three from this session, plus Maher et al. (1996) and Gero and Kazakov (1996)). All five of these papers focussed on methods of searching the spaces, and further, all five used non-systematic methods to carry out that search. Although conclusions should not be drawn from such a small sample, it would appear to indicate a development in the field, in that the research is now building on a framework laid down earlier, and considering how that framework can be used in practice.

\section{Conclusion}

Very little consensus was reached during the discussion. One point worthy of note was the acceptance by almost all present of the idea of exploration being a better description of the design process than pure search. Other than that, the discussion served to highlight that there are many questions which remain unanswered, both in the general area of relating the design process to generation, and in specific issues in the control of the search and generation process. 


\section{Acknowledgments}

I would like to thank Stephan Rudolph, for providing the clear and comprehensive notes on which this summary is based, and Mary Lou Maher, who chaired the discussion and gave it its structure by posing the three questions to be discussed. Jon Cagan provided a number of helpful comments on an earlier draft. Finally, I must thank all the participants of the workshop, who were responsible for most of the ideas raised in this summary; I apologize for not providing individual attributions.

\section{References}

Andersson, K.: 1994, A vocabulary for conceptual design, in J. S. Gero and E. Tyugu (eds), Formal Design Methods for CAD, North-Holland, Amsterdam, pp. 157-171.

Brown, K. N., McMahon, C. A. and Sims Williams, J. H.: 1994, A formal language for the design of manufacturable objects, in J. S. Gero and E. Tyugu (eds), Formal Design Methods for CAD, North-Holland, Amsterdam, pp 135-155.

Brown, K. N. and Cagan, J.: 1996, Modified shape annealing for optimally-directed generation: Initial results, in J. S. Gero (ed.), Advances in Formal Design Methods for $C A D$, Chapman \& Hall, London, pp. 59-73.

Cagan, J., and Mitchell, W. J.: (1994, A grammatical approach to network flow synthesis, in J. S. Gero and Tyugu, E. (eds), Formal Design Methods for CAD, North-Holland, Amsterdam, pp. 173-189.

Carlson, C.: 1994, Design space description formalisms, in J. S. Gero and Tyugu, E. (eds), Formal Design Methods for CAD, North-Holland, Amsterdam, pp. 121-131.

Gero, J. S. and Kazakov, V. A.: 1996, Evolving building blocks for design using genetic engineering: A formal approach in J. S. Gero (ed.), Advances in Formal Design Methods for $C A D$, Chapman \& Hall, London, pp. 31-50.

Gero, J. S. and Tyugu, E. (eds): 1994, Formal Design Methods for CAD, North-Holland, Amsterdam.

Heisserman, J. and Woodbury, R.: 1994, Geometric design with boundary solid grammars, in J. S. Gero and Tyugu, E. (eds), Formal Design Methods for CAD, North-Holland, Amsterdam, pp. 85-105.

Kirkpatrick, S., Gelatt, C. D. Jr and Vecchi, M. P.: 1983, Optimization by simulated annealing, Science, 220(4598), 671-679.

Maher, M. L., Poon, J. and Boulanger, S.: 1996, Formalizing design exploration as coevolution: a combined gene approach, in J. S. Gero (ed.), Advances in Formal Design Methods for CAD, Chapman \& Hall, London, pp. 3-30.

Simon, H.: 1967, The Sciences of the Artificial, MIT Press, Cambridge, MA.

Shih, S.-G. and Schmitt, G.: 1994, The use of post interpretation for grammar-based generative systems, in J. S. Gero and Tyugu, E. (eds), Formal Design Methods for CAD, North-Holland, Amsterdam, pp. 107-120.

Smithers, T., Corne, D. and Ross, P.: 1994, On computing exploration and solving design problems, in J. S. Gero and Tyugu, E. (eds), Formal Design Methods for CAD, NorthHolland, Amsterdam, pp 293-313.

Zozaya-Gorstiza, C. and Estrada, L. F.: 1996, Incorporating heuristics and a meta-architecture in a genetic algorithm for harness design, in J. S. Gero (ed.), Advances in Formal Design Methods for CAD, Chapman \& Hall, London, pp. 75-96. 